\title{
Effects of a cervical disc prosthesis on segmental and cervical spine alignment
}

\author{
Gwynedd E. Pickett, M.D., F.R.C.S.(C), Demytra K. Mitsis, Lali H. Sekhon, M.B.B.S., \\ Ph.D., F.R.A.C.S., WiLliam R. SEARS, M.B.B.S., F.R.A.C.S., \\ AND NeIL Duggal, M.D., M.Sc., F.R.C.S.(C)
}

Division of Neurosurgery, Department of Clinical Neurological Sciences, London Health Sciences Centre, London, Ontario, Canada; and Department of Neurosurgery and Spinal Injuries Unit, Royal North Shore Hospital, University of Sydney, Australia

\begin{abstract}
Object. Cervical arthroplasty offers the promise of maintaining motion of the functional spinal unit (FSU) after anterior cervical discectomy. The impact of cervical arthroplasty on sagittal alignment of the FSU needs to be addressed, together with its effect on overall sagittal balance of the cervical spine.

Methods. The authors prospectively reviewed radiographic and clinical outcomes in 14 patients who received the Bryan Cervical Disc prosthesis (Medtronic Sofamor Danek, Memphis, TN), for whom early ( $<6$ months) and late (6-24 months) follow-up data were available. Static and dynamic radiographs were measured by hand and computer to determine the angles formed by the endplates of the natural disc preoperatively, those formed by the shells of the implanted prosthesis, the angle of the FSU, and the C2-7 Cobb angle. The range of motion (ROM) was also determined radiographically, whereas clinical outcomes were assessed using the Neck Disability Index (NDI), and Short Form-36 (SF-36) questionnaires.

The ROM was preserved following surgery, with a mean preoperative sagittal rotation angle of $8.96^{\circ}$, which was not significantly different from the late postoperative value of $8.25^{\circ}$. When compared with the preoperative disc space angle, the shell endplate angle in the neutral position became kyphotic in the early and late postoperative periods (mean change $-3.8^{\circ}$ in the late follow-up period; $\left.\mathrm{p}=0.0035\right)$. The FSU angles also became significantly more kyphotic postoperatively, with a mean change of $-6^{\circ}(\mathrm{p}=0.0006)$. The Cobb angles varied widely preoperatively and did not change significantly after surgery. There was no statistical correlation between the NDI and SF-36 outcomes and cervical kyphosis.

Conclusions. Cervical arthroplasty preserves motion of the FSU. Both the endplate angle of the treated disc space and the angle of the FSU became kyphotic after insertion of the Bryan prosthesis. The overall sagittal balance of the cervical spine, however, was preserved.
\end{abstract}

\section{KEY WORDS • cervical arthroplasty • sagittal balance • spinal alignment • artificial disc • cervical kyphosis}

Cervical arthroplasty is an emerging field that offers the promise of restoring normal spinal motion after anterior cervical discectomy. The current standard practice of anterior cervical fusion provides excellent symptomatic relief in the short term, ${ }^{3,5,18,20,21}$ but has been implicated in abnormal kinematic strain on adjacent spinal levels., ${ }^{43}$ This is believed to result in more rapid progression of degenerative disease and/or mechanical instability at these adjacent levels. ${ }^{2,9,13,22,24}$ The goal of spinal arthroplasty is to reduce or eliminate adjacent-segment disease by preserving motion at the treated level. The ROM, however, is only a single aspect of spinal biomechanics that is affected following arthroplasty. The effect of the prosthesis on sagittal alignment of both the treated level and the whole cervical

Abbreviations used in this paper: $\mathrm{FSU}=$ functional spinal unit; NDI = Neck Disability Index; ROM = range of motion; SF-36 = Short Form-36; VB = vertebral body. spine must be considered. Kyphotic deformity following anterior cervical discectomy and fusion has been implicated in the development of segmental instability, clinically significant adjacent-segment disease, and poor functional recovery. ${ }^{6,10,12}$ We describe the radiographic and clinical impact of the Bryan Cervical Disc on the FSU and overall sagittal balance of the cervical spine after spinal arthroplasty.

\section{CLINICAL MATERIAL AND METHODS}

\section{Patient Population}

In this prospective cohort study we enrolled 14 consecutive patients who had undergone implantation of the Bryan Cervical Disc prosthesis (Medtronic Sofamor Danek, Memphis, TN) at C5-6 and/or C6-7 and in whom at least 6 months of clinical and radiographic follow-up review had been completed. All patients had presented with ra- 
diculopathy and/or myelopathy, and all of them underwent anterior cervical discectomy followed by implantation of the disc prosthesis. The study group consisted of five women and nine men whose mean age was 41.8 years (range 30-56 years). The duration of preoperative symptoms ranged from 1 to 60 months (mean duration 12.4 months). Twelve of the 14 patients presented with radiculopathy and two with myelopathy. In patients with radiculopathy, pain in the upper extremity constituted the majority of their symptoms. Neck pain constituted less than $30 \%$ of any patient's preoperative pain syndrome.

\section{Surgical Procedure}

The surgical procedure has been described previously. ${ }^{14}$ The calculated angle of endplate preparation and disc insertion were as outlined in the product monograph in the first 10 patients. In the remaining patients, the angle of insertion was determined by placing a line parallel to the angle of the disc space and using the angle formed by a perpendicular to this line relative to the plumb line. Static and dynamic digital radiographs were obtained preoperatively and at $1.5,3,6,12$, and 24 months postoperatively. Clinical outcomes were assessed at each time point by patients' self-administration of the NDI and SF36 health questionnaires.

Radiographs were analyzed to determine the shell angle, defined as the angle of the disc space formed preoperatively by the natural endplates and postoperatively by the shells of the prosthesis. The FSU angle was defined as that formed by lines drawn at the superior margin of the superior VB defining the disc space and the inferior margin of the inferior body (Fig. 1). The Cobb angles from C2-7 were also determined. Angles were measured using quantitative measurement analysis software (Medical Metrics, Inc., Houston, TX), which uses extrapolative algorithms to calculate the intersecting angle between two lines drawn by the investigator. All angles were measured five times and a mean angle was determined, to allow for investigator error in assessing the margins of the VBs. The same investigator produced all computerized measurements. Hand measurements of the shell angles were also

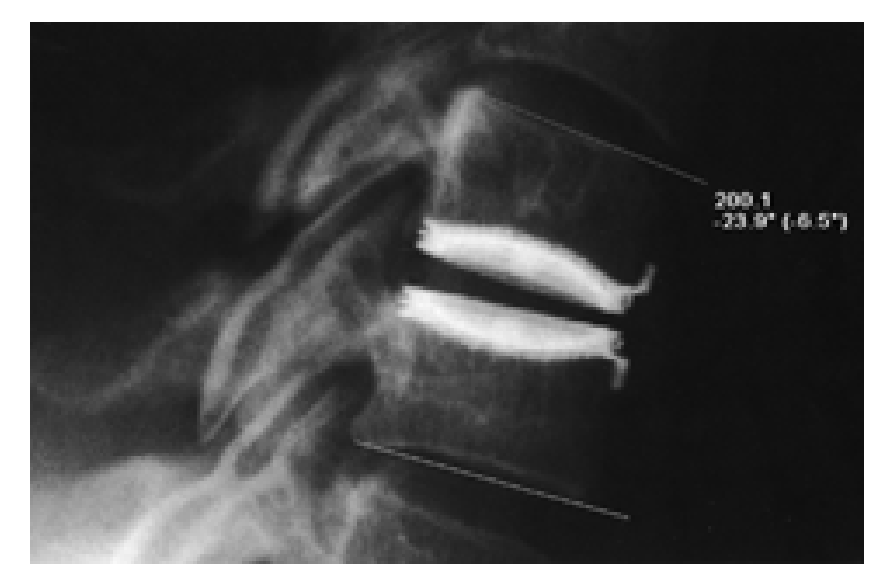

Fig. 1. Photograph showing the screen from the quantitative measurement analysis software, which demonstrates the angle of the FSU. The number in parentheses represents the angle between the two lines $\left(^{\circ}\right)$. performed and compared with computer-produced measurements. Because hand- and computer-measured values were not significantly different, those obtained with the computer were used. We chose to define the late followup period as 6 months or later; we had found in a separate pilot study that there was no significant difference in biomechanical parameters and angle calculations between 6 and 24 months of follow up (unpublished data).

\section{RESULTS}

Nine discs were placed at C5-6 and six at C6-7 (in one patient discs were implanted at both levels). No complications occurred at the time of surgery. Postoperatively, one patient experienced transient unilateral vocal cord paralysis that resolved by 6 weeks. All other patients had an uneventful postoperative course and were discharged home the day after surgery. No patient has been lost to follow up. The duration of follow up ranged from 6 to 24 months (mean 12 months). Seven patients held jobs preoperatively, five were receiving disability income, one was receiving worker's compensation, and one was a homemaker. Only one patient was still dependent on disability income after surgery. All previously working patients returned to work postoperatively, as did the patient who was receiving Workers' Compensation and also four of the five who were receiving disability.

\section{Radiographic Analysis}

Range of Motion. Motion was preserved in all cases. The mean postoperative ROM produced by the prosthesis was $7.14^{\circ}$ in early follow-up evaluations and $8.25^{\circ}$ in late follow up. These values were not significantly different from the preoperative one of $8.96^{\circ}$ (Student t-test, $\mathrm{p}=$ 0.152 and 0.639 , respectively, in early compared with late follow up).

Disc Space Angle. Computer-measured values for the disc space (endplate) angle in neutral, flexion, and extension positions are shown in Table 1 . Twelve patients had a lordotic disc space angle at the treated segment before surgery, whereas the other two had a straight segment with less than $1^{\circ}$ of angulation. Postoperatively, all patients demonstrated varying degrees of kyphosis of the prosthesis shells at the treated level. The mean postoperative disc space angle in the neutral position was $-6.07^{\circ}$ in early follow-up assessments, which was significantly different from the mean preoperative value of $4.7^{\circ}$ (paired Student t-test, $p=0.0002$ ). Late follow-up evaluations did not demonstrate significant improvement, with a mean shell angle of $-5.39^{\circ}(p=0.0035$ compared with the mean preoperative angle).

The FSU Angle. The FSU angles became more kyphotic postoperatively (Table 2 ). Seven patients demonstrated segmental kyphosis, with an FSU angle less than $0^{\circ}$. The mean change for the cohort was $-6^{\circ}$. Most of the change occurred between preoperative and early follow-up assessments, with little change between early and late follow-up review.

Overall Cervical Alignment. The Cobb angles varied widely preoperatively, from $30.2^{\circ}$ of lordosis to $17.2^{\circ}$ of kyphosis (Table 2). There was no significant change in the 
TABLE 1

Shell angles $\left({ }^{\circ}\right)$ measured on lateral radiographs in 14 patients who underwent placement of cervical disc prostheses*

\begin{tabular}{|c|c|c|c|c|c|c|c|c|c|c|c|c|}
\hline $\begin{array}{l}\text { Treated } \\
\text { Level }\end{array}$ & \multicolumn{4}{|c|}{ Preop } & \multicolumn{4}{|c|}{ Early (1.5-3 mos) } & \multicolumn{4}{|c|}{ Late (6-24 mos) } \\
\hline C5-6 & 6.0 & 2.5 & -4.7 & 10.7 & -3.3 & -5.2 & -16.9 & 13.6 & -4.3 & -7.2 & -11.8 & 7.5 \\
\hline C5-6 & 6.0 & 3.1 & -8.7 & 14.7 & -3.0 & -7.2 & -15.0 & 12.0 & -3.8 & -10.3 & -16.2 & 12.4 \\
\hline C5-6 & 10.6 & 7.7 & 7.3 & 3.3 & -9.1 & -9.6 & -10.1 & 1.0 & -7.5 & -9.3 & -16.1 & 8.6 \\
\hline C5-6 & 3.5 & 3.2 & -4.5 & 8.0 & -6.6 & -7.0 & -18.0 & 11.4 & -4.3 & -7.2 & -11.8 & 7.5 \\
\hline C5-6 & 14.4 & 11.6 & 2.1 & 12.3 & -3.4 & -6.6 & -13.4 & 10.0 & 0.7 & -4.2 & -12.6 & 13.3 \\
\hline C6-7 & 2.5 & 1.7 & -12.0 & 14.5 & -12.3 & -13.2 & -16.3 & 4.0 & -13.6 & -16.0 & -17.1 & 3.5 \\
\hline C6-7 & 8.8 & 7.8 & 2.3 & 6.5 & -0.6 & -4.4 & -3.7 & 3.1 & 1.5 & -1.1 & -7.8 & 9.3 \\
\hline C6-7 & 6.0 & 4.6 & -6.2 & 12.2 & -5.7 & -6.8 & -11.4 & 5.7 & -6.5 & -6.1 & -11.8 & 5.3 \\
\hline C6-7 & 5.4 & 3.9 & 3.8 & 1.6 & -4.4 & -4.9 & -13.0 & 8.4 & 0.2 & -1.5 & -7.9 & 8.1 \\
\hline C6-7 & 7.7 & 6.1 & -1.2 & 8.9 & -2.6 & -10.7 & -3.0 & 8.1 & -1.6 & -2.4 & -9.1 & 7.5 \\
\hline C6-7 & 7.5 & 0.5 & -1.3 & 8.8 & -2.1 & -5.3 & -9.3 & 7.2 & -1.1 & -4.0 & -8.5 & 7.4 \\
\hline
\end{tabular}

* Ext = extension; Flex = flexion; $\mathrm{Neu}=$ neutral; $\mathrm{SD}=$ standard deviation

$\dagger$ Total ROM from a maximally flexed to maximally extended position.

mean Cobb angles postoperatively in early or late followup findings (paired Student t-test, $\mathrm{p}=0.28$ and 0.27 for early and late follow-up assessments, respectively). In eight patients with lordotic alignment preoperatively (defined as $\geq 10^{\circ}$ ) the spinal alignment remained lordotic postoperatively. Of two patients with a straight preoperative alignment (defined as $0-10^{\circ}$ ), the alignment in one became slightly kyphotic, whereas the other was lordotic

TABLE 2

The FSU and Cobb angles $\left({ }^{\circ}\right)$ in 14 patients with cervical disc prostheses measured in the neutral lateral position*

\begin{tabular}{|c|c|c|c|c|c|c|}
\hline \multirow{2}{*}{$\begin{array}{c}\text { Treated } \\
\text { Level }\end{array}$} & \multicolumn{3}{|c|}{ FSU Angle } & \multicolumn{3}{|c|}{ Cobb Angle (C2-7) } \\
\hline & Preop & Early & Late & Preop & Early & Late \\
\hline C5-6 & -5.6 & -6.9 & -7.2 & 45.2 & -0.9 & -4.3 \\
\hline C5-6 & -0.7 & -3.6 & -4.6 & 16.8 & 11.6 & 8.0 \\
\hline C5-6 & 6.2 & 1.7 & 1.3 & - & - & 13.9 \\
\hline C5-6 & 7.0 & -1.25 & 0.8 & 30.2 & 11.9 & 19.6 \\
\hline C5-6 & 1.4 & 2.7 & 2.2 & -1.1 & 4.4 & 2.0 \\
\hline C5-6 & 7.4 & 2.7 & 3.5 & 23.8 & 13.0 & 16.0 \\
\hline C5-6 & -0.6 & -1.1 & -1.4 & 13.7 & 19.2 & 33.7 \\
\hline C5-6 & 5.8 & -7.0 & -7.5 & 10.0 & 7.4 & 3.3 \\
\hline C5-7 & 15.9 & 10.6 & 9.6 & 28.5 & 19.1 & 19.0 \\
\hline C6-7 & 1.4 & 0.5 & -1.7 & -17.8 & -15.6 & -19.8 \\
\hline C6-7 & 4.8 & -1.5 & -1.8 & 20.3 & 21.3 & 22.8 \\
\hline C6-7 & 12.8 & 2.5 & -0.5 & 10.1 & 9.6 & -14.0 \\
\hline C6-7 & - & 3.2 & 5.4 & - & 37.3 & 28.7 \\
\hline C6-7 & 4.3 & -13.9 & -13.5 & 6.7 & 14.6 & 16.1 \\
\hline \multicolumn{7}{|l|}{ all levels } \\
\hline mean & 4.62 & -0.81 & -1.41 & 12.20 & 11.76 & 10.36 \\
\hline $\mathrm{SD}$ & 5.72 & 5.85 & 5.77 & 13.41 & 12.41 & 15.48 \\
\hline \multicolumn{7}{|l|}{ C5-6 } \\
\hline mean & 2.61 & -1.59 & -2.16 & 14.09 & 9.51 & 11.53 \\
\hline $\mathrm{SD}$ & 4.71 & 3.95 & 4.02 & 10.71 & 6.52 & 11.98 \\
\hline \multicolumn{7}{|l|}{ C6-7 } \\
\hline mean & 5.83 & -1.84 & -2.42 & 4.83 & 13.44 & 6.76 \\
\hline $\mathrm{SD}$ & 4.89 & 6.99 & 6.86 & 16.15 & 19.30 & 22.15 \\
\hline
\end{tabular}

* $=$ not performed. in late follow-up evaluations. Two patients exhibited kyphotic alignment (defined as $<0^{\circ}$ ) preoperatively: in one who had minimal kyphosis the alignment was considered straight in a late follow-up evaluation, whereas the frankly kyphotic spine in the other $\left(-17.2^{\circ}\right)$ remained so. In two patients, Cobb angles could not be determined because we were unable to visualize the inferior margin of the C-7 VB. Imaging performed on the 1st postoperative day to check prosthesis positioning indicated a tendency for the Cobb angle to become more kyphotic in the immediate postoperative period, but it returned to its usual state by 6 weeks.

\section{Clinical Outcome}

The NDI scores decreased significantly in both early and late follow-up evaluations (paired Student t-test, $\mathrm{p}=$ 0.038 and 0.006 for early and late findings, respectively), from a mean preoperative score of 20.1 , to 11.1 at 3 months and 7.4 at 1 year (Fig. 2). No significant difference in postoperative NDI scores was identified between patients with segmental kyphosis or lordosis. Nevertheless, the one patient with substantial pre- and postoperative global cervical kyphosis reported severe axial neck pain and continued to have an NDI score of 30, indicating severe disability, in late follow-up evaluations.

The SF-36 scores (Fig. 3) demonstrated significant improvement in late follow-up results in the areas of physical function $(\mathrm{p}=0.002)$, physical role $(\mathrm{p}=0.017)$, body pain $(\mathrm{p}=0.008)$, vitality $(\mathrm{p}=0.002)$, and emotional role ( $p=0.0004$, all according to the paired Student t-test). Physical and mental component summary scores showed a trend toward improvement, which did not reach significance in this small group $(p=0.084$ and 0.076 for physical and mental components, respectively). The mean preoperative physical component score was 34.3 , which improved to 45.1 in the late follow-up period, whereas the mean preoperative mental component score was 44.1, and this improved to 50.9 in the late follow-up period. 


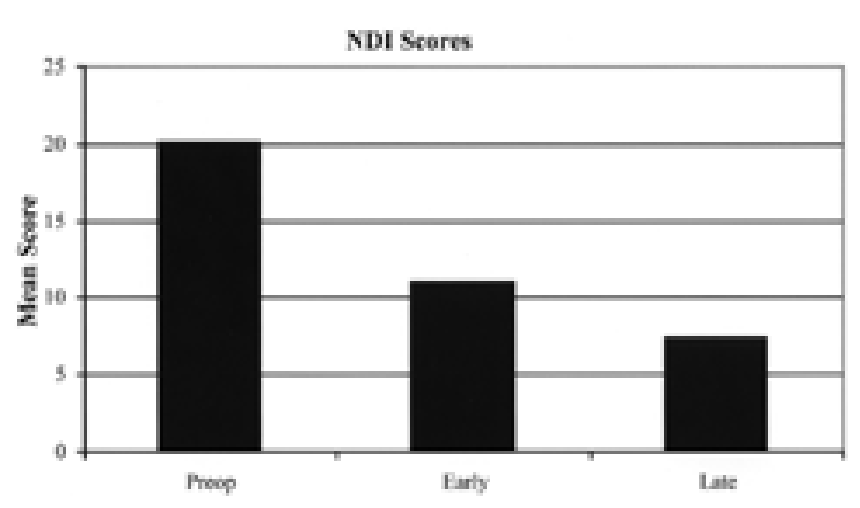

Fig. 2. Bar graph showing the mean NDI scores preoperatively and at 3,6, and 12 months postoperatively (questionnaire scored from $0-50$ ). Scores were significantly improved in both early and late follow-up evaluations.

\section{DISCUSSION}

It is controversial whether cervical kyphosis influences clinical outcome after anterior cervical discectomy with or without fusion. ${ }^{1,11}$ In several studies researchers have examined the impact of surgery on sagittal alignment of the cervical spine..$^{1,11,17,19,23}$ Cervical arthroplasty, a recent advance in spinal instrumentation, promises to expand the surgical armamentarium for the management of degenerative disc disease. Early clinical results from European trials of single- and two-level arthroplasty in which the Bryan disc was used have included outcomes of excellent, good, or fair in more than $90 \%$ of patients at 1 year. $^{7}$ The ROM was reportedly preserved in $88 \%$ of patients with single-level and $86 \%$ of those with two-level prostheses at 1 year. Nevertheless, although the ROM is an important feature of an artificial disc, it is only a single measure of spinal biomechanics. The effect of the artificial disc on angulation at the treated level and on the overall spinal alignment may be important to long-term clinical outcomes and rates of adjacent-segment disease.

Our results demonstrated that the endplate or shell angle of the Bryan Cervical Disc assumed a kyphotic angulation postsurgery (mean change $-8.2^{\circ}$ ), and that this re-

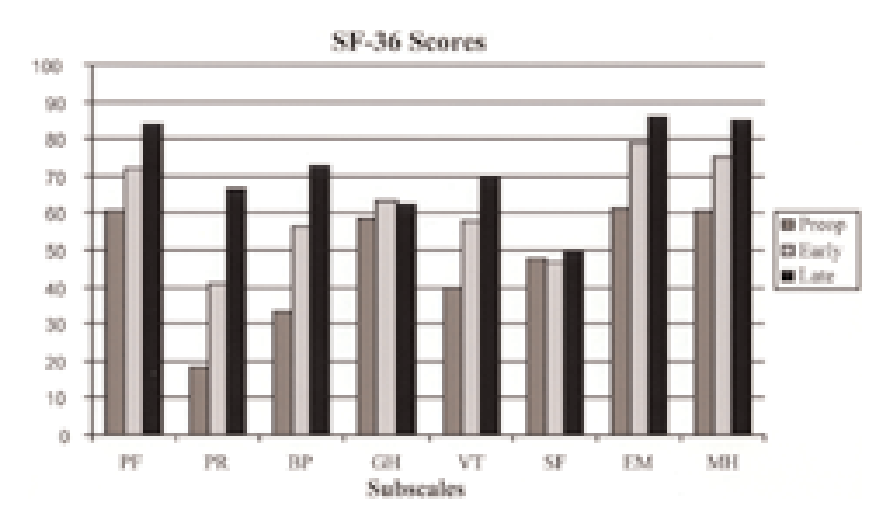

Fig. 3. Bar graph showing the mean SF-36 scores in eight categories: $\mathrm{BP}=$ body pain; $\mathrm{EM}=$ emotional role; $\mathrm{GH}=$ general health; $\mathrm{MH}=$ mental health; $\mathrm{PF}=$ physical function; $\mathrm{PR}=$ physical role; $\mathrm{SF}=$ social function; $\mathrm{VT}=$ vitality . sulted in kyphosis of the FSU. Despite the kyphosis seen at the level of the endplates of the disc, the overall alignment between C-2 and C-7 did not change significantly, indicating that the untreated segments of the cervical spine were compensating for a focal kyphosis. Kyphotic angulation in the neutral position did not prevent the disc from providing sagittal plane motion (also referred to as ROM) equivalent to the preoperative ROM.

With most of the available disc replacement systems, including the Bryan disc, the angle of disc insertion can significantly alter the orientation of the prosthesis. This is related to bone removal and endplate preparation for the prosthesis. The angle of disc insertion is arbitrary, with no precise measure available to predict accurately the impact of the prosthesis on the sagittal alignment. A number of different methods have been used to calculate the angle of insertion for the Bryan disc. In its description of the surgical technique for the Bryan Cervical Disc system, the product monograph suggests that the disc space angle can be calculated by measuring the angle between the plumb line and a line connecting the posterior inferior corner of the caudal VB and the posterior superior corner of the cephalad VB. The results obtained using this method can be quite variable, depending on the level of the target disc space and the method of intraoperative positioning. Furthermore, with this method of angle calculation, the angle of insertion is determined when the patient is supine with muscles relaxed from anesthesia. Again, this may not represent the true cervical alignment.

In another method used to calculate the angle of insertion, an attempt is made to place the prosthesis in parallel with the angle of the disc space. Because of the passive nature of the prostheses, this strategy may minimize the risk of introducing kyphosis into the cervical spine. Changing the angle of disc insertion, however, is only effective in avoiding kyphosis in the prosthesis endplates. Unfortunately, one cannot correct an underlying straight or kyphotic cervical spine by changing only the angle of prosthesis insertion. Because in the majority of our patients the angle of disc insertion was that suggested by the product monograph, our results for kyphotic endplate and FSU angles may be technique- and/or surgeon-related. Further studies will be needed to address the issue of how to calculate the angle of disc insertion reliably.

The Cobb angle from $\mathrm{C} 2-7$ did not change significantly in the early or late postoperative period when compared with preoperative imaging. Neuroimaging studies performed on the 1st postoperative day indicated a tendency for the cervical spine alignment to become slightly more kyphotic immediately postoperatively, but it returns to the preoperative state by 6 weeks. This finding may reflect neck pain as an early response to surgery. We hypothesize that the preoperative global spinal alignment, as assessed by determining the Cobb angle from C2-7, may influence the observed shell angles. It should be noted that Cobb angles compare only the ends of the cervical curve, but do not describe what happens to the curve internally, as in segmental kyphosis. ${ }^{8}$ Sekhon ${ }^{16}$ attained some degree of correction of alignment in two of seven patients who suffered "loss of lordosis" preoperatively. This would not be predicted by the biomechanical parameters of the disc, and we speculate that it may relate to relief of pain and 
relaxation of spinal musculature, as was observed between the early and late postoperative visits in our patients.

Eight of our patients had cervical lordosis of $10^{\circ}$ or more preoperatively; two had C2-7 Cobb angles between 0 and $10^{\circ}$; two had kyphotic spines (Cobb angle $<0^{\circ}$ ); and the Cobb angle could not be determined in the other two patients. In this small group we could not define a statistically clear relationship between the preoperative Cobb angle and postoperative alignment. Nevertheless, based on our observations pertaining to insertion technique and the disc kinematics, we propose three categories. In patients with normal preoperative lordosis, alignment is typically preserved, even if the prosthesis shells develop a kyphotic angulation. Patients with preoperative straightening of the cervical spine may have a preserved alignment after disc insertion, but are at risk for development of segmental kyphosis if the angle of disc insertion is not correct. In our limited experience, patients with a preoperative kyphosis may remain unchanged or they may experience worsening of the kyphosis. The prosthesis is passive, and hence one would expect that it would be unable to restore lordosis to a spine that has focal or global kyphosis. An artificial disc inserted into a kyphotic segment is likely to take on the angle and local biomechanics determined by the preoperative diseased FSU. The Bryan Cervical Disc is not designed to correct kyphosis.

\section{Effect of Cervical Kyphosis}

Although the artificial cervical disc is new, the problem of segmental kyphosis and its effects on the adjacent levels and sagittal spinal alignment is not. The numerous papers in the literature pertaining to the question of whether to fuse after anterior cervical discectomy often return to the question of alignment. ${ }^{11,15,17,20,23}$ Proponents of fusion note that insertion of a bone plug, whether or not supplemented by anterior plate fixation, is the sole method available to restore or preserve cervical lordosis. Segmental kyphosis is significantly more common after anterior cervical discectomy without arthrodesis. ${ }^{1}$

In a retrospective study of 42 patients followed for a mean of 10 years after undergoing anterior cervical discectomy and fusion for symptomatic cervical spondylosis, Katsuura, et al., ${ }^{11}$ found that degeneration of adjacent levels was significantly associated with loss of physiological cervical lordosis. They speculated that fusion in kyphosis loads posterior slipping forces onto adjacent vertebral levels and may cause higher loads on the posterior column than a fusion in lordosis. The implication is that physiological lordotic fixation is required for prevention of neurological deterioration. Because artificial discs by definition are not intended to produce fixation, the question becomes whether the spine will tolerate kyphosis as long as motion is preserved.

Troyanovich, et al., ${ }^{19}$ performed a retrospective analysis of intersegmental and global sagittal alignment in 57 patients who had undergone single-level anterior cervical discectomy and fusion with or without anterior plating. They reported that the segmental angle became on average $2.5^{\circ}$ more kyphotic in the group that did not receive plates, whereas the group in which plates were implanted gained $5.67^{\circ}$ of lordosis. Interestingly, however, the over- all cervical lordosis did not change significantly, indicating that compensation occurs at other levels. Our results similarly showed that Cobb angles from C2-7 did not change significantly in the postoperative period, regardless of changes in the endplate or segmental angles.

In patients with preoperative focal kyphosis, there is a risk that the kyphosis may worsen after insertion of the disc. This may represent progression of their disease at this level, rather than a causal relationship to the prosthesis. The disc preserves the existing motion of the spine, which has been altered by the underlying degenerative process: it thus maintains an abnormal condition rather than correcting it. Careful patient selection and attention to the angle of insertion should reduce the risk of postoperative kyphotic deformity and its attendant problems.

\section{CONCLUSIONS}

Kyphosis of the endplate shells of an implanted Bryan Cervical Disc prosthesis is common, and it is likely to be related to angle of disc insertion and patient selection. This produces focal segmental kyphosis, although global cervical alignment is preserved. The ROM is maintained and neck disability is significantly reduced following insertion of the artificial disc. Future studies will need to address whether postoperative cervical alignment is associated with clinical outcomes in patients undergoing cervical arthroplasty.

\section{References}

1. Abd-Alrahman N, Dokmak AS, Abou-Madawi A: Anterior cervical discectomy (ACD) versus anterior cervical fusion (ACF), clinical and radiological outcome study. Acta Neurochir 141: 1089-1092, 1999

2. Baba H, Furusawa N, Imura S, et al: Late radiographic findings after anterior cervical fusion for spondylotic myeloradiculopathy. Spine 18:2167-2173, 1993

3. Connolly PJ, Esses SI, Kostuik JP: Anterior cervical fusion: outcome analysis of patients fused with and without anterior cervical plates. J Spinal Disord 9:202-206, 1996

4. Eck JC, Humphreys SC, Lim TH, et al: Biomechanical study on the effect of cervical spine fusion on adjacent-level intradiscal pressure and segmental motion. Spine 27:2431-2434, 2002

5. Epstein NE: Anterior cervical diskectomy and fusion without plate instrumentation in 178 patients. J Spinal Disord 13:1-8, 2000

6. Ferch RD, Shad A, Cadoux-Hudson TA, et al: Anterior correction of cervical kyphotic deformity: effects on myelopathy, neck pain, and sagittal alignment. J Neurosurg (Spine 1) 100: 13-19, 2004

7. Goffin J, Van Calenbergh F, van Loon J, et al: Intermediate follow-up after treatment of degenerative disc disease with the Bryan Cervical Disc Prosthesis: single-level and bi-level. Spine 28:2673-2678, 2003

8. Harrison DE, Harrison DD, Cailliet R, et al: Cobb method or Harrison posterior tangent method: which to choose for lateral cervical radiographic analysis? Spine 25:2072-2078, 2000

9. Hilibrand AS, Carlson GD, Palumbo MA, et al: Radiculopathy and myelopathy at segments adjacent to the site of a previous anterior cervical arthrodesis. J Bone Joint Surg Am 81: 519-528, 1999

10. Katsuura A, Hukuda S, Imanaka T, et al: Anterior cervical plate used in degenerative disease can maintain cervical lordosis. J Spinal Disord 9:470-476, 1996

11. Katsuura A, Hukuda S, Saruhashi Y, et al: Kyphotic malalign- 
ment after anterior cervical fusion is one of the factors promoting the degenerative process in adjacent intervertebral levels. Eur Spine J 10:320-324, 2001

12. Kawakami M, Tamaki T, Iwasaki H, et al: A comparative study of surgical approaches for cervical compressive myelopathy. Clin Orthop 381:129-136, 2000

13. Matsunaga S, Kabayama S, Yamamoto T, et al: Strain on intervertebral discs after anterior cervical decompression and fusion. Spine 24:670-675, 1999

14. Pickett GE, Duggal N: Artificial disc insertion following anterior cervical discectomy. Can J Neurol Sci 30:278-283, 2003

15. Savolainen S, Rinne J, Hernesniemi J: A prospective randomized study of anterior single-level cervical disc operations with long-term follow-up: surgical fusion is unnecessary. Neurosurgery 43:51-55, 1998

16. Sekhon LHS: Cervical arthroplasty in the management of spondylotic myelopathy. J Spinal Disord Tech 16:307-313, 2003

17. Sonntag VK, Klara P. Controversy in spine care. Is fusion necessary after anterior cervical discectomy? Spine 21: 1111-1113, 1996

18. Thorell W, Cooper J, Hellbusch L, et al: The long-term clinical outcome of patients undergoing anterior cervical discectomy with and without intervertebral bone graft placement. Neurosurgery 43:268-273, 1998

19. Troyanovich SJ, Stroink AR, Kattner KA, et al: Does anterior plating maintain cervical lordosis versus conventional fusion techniques? A retrospective analysis of patients receiving single-level fusions. J Spinal Disord Tech 15:69-74, 2002

20. Wang JC, McDonough PW, Endow K, et al: The effect of cervical plating on single-level anterior cervical discectomy and fusion. J Spinal Disord 12:467-471, 1999

21. Watters WC III, Levinthal R: Anterior cervical discectomy with and without fusion. Results, complications, and long-term follow-up. Spine 19:2343-2347, 1994

22. Wigfield C, Gill S, Nelson R, et al: Influence of an artificial cervical joint compared with fusion on adjacent-level motion in the treatment of degenerative cervical disc disease. J Neurosurg (Spine 1) 96:17-21, 2002

23. Yamamoto I, Ikeda A, Shibuya N, et al: Clinical long-term results of anterior discectomy without interbody fusion for cervical disc disease. Spine 16:272-279, 1991

24. Yonenobu K, Okada K, Fuji T, et al: Causes of neurologic deterioration following surgical treatment of cervical myelopathy. Spine 11:818-823, 1986

Manuscript received July 16, 2004.

Accepted in final form August 16, 2004.

Address reprint requests to: Neil Duggal, M.D., Division of Neurosurgery, Department of Clinical Neurological Sciences, London Health Sciences Centre, 339 Windermere Road, London, Ontario, Canada N6A 5A5. 